\title{
EXTENSION OF BAKER'S ANALOGUE OF LITTLEWOOD'S DIOPHANTINE APPROXIMATION PROBLEM
}

\author{
BY TAKaO KOMATSU
}

\section{Introduction.}

The famous but still unsolved problem of Littlewood can be stated as follows: for each pair of real numbers $\theta$ and $\phi$ and each $\varepsilon>0$, does there exist a positive integer $n$ such that

$$
n\|n \theta\|\|n \phi\|<\varepsilon \text { ? }
$$

Here $\|\alpha\|$ denotes the difference between $\alpha$ and the nearest integer. In 1963 Davenport and Lewis [1] obtained a negative answer for an analogous question concerning formal power series. The following year Baker [2] gave examples where the construction of Davenport and Lewis holds. And as a generalization of these results, he indicated the following result:

THEOREM (Baker (1964)). If $\lambda_{1}, \cdots, \lambda_{r}$ are distinct real numbers, none of them 0 , and $u(t), u_{1}(t), \cdots, u_{r}(t)$ are real polynomials with $u(t) \neq 0$, then

$$
|u(t)|_{K} \prod_{j=1}^{r}\left|u_{j}(t)-e^{\lambda_{j} / t} u(t)\right|_{K} \geqq e^{-R}
$$

where $R=(1 / 2)\left(r^{3}+r\right)$.

The valuation of a formal power series relative to the real number field $K$ is defined by

$$
\left|a_{m} t^{m}+a_{m-1} t^{m-1}+\cdots\right|_{K}=e^{m} \quad\left(a_{m} \neq 0, m \text { is integer }\right) .
$$

The purpose of this paper is an extension of Baker's result, proving the following theorem :

THEOREM. Let $n, r$ be positive integers. If $\lambda_{1}, \cdots, \lambda_{r}$ are distinct real numbers, none of them 0 , and $u(t), u_{1}(t), \cdots, u_{r}(t)$ are real polynomials with $u(t) \neq 0$, then

$$
|u(t)|_{K} \prod_{j=1}^{r}\left|u_{j}(t)-e^{\lambda_{j} / t^{n}} u(t)\right|_{K} \geqq e^{-R(n, r)},
$$

Received November 10, 1990. 
where $R(n, r)=(1 / 2) n\left(r^{3}+r\right)$.

\section{The construction of polynomials}

Let $m_{j}(j=1,2, \cdots, r)$ be positive integers. Then clearly there exist real polynomials $P_{0}(x), P_{0}^{(1)}(x), \cdots, P_{0}^{(r)}(x)$ of degree at most $h=\sum_{j=1}^{r} m_{j}-r$, not all identically zero, such that for $j=1,2, \cdots, r$

$$
P_{0}^{(j)}(x)-e^{\lambda_{j} x} P_{0}(x)=b_{m_{j+h}^{(j)}}^{m_{j^{+h}}}+b_{m_{j^{+h+1}}^{(j)}} x^{m_{j+h+1}}+\cdots,
$$

where $(j)$ in $P_{0}^{(j)}(x)$ denotes the suffix. $P_{0}(x), P_{0}^{(j)}(x)(j=1,2, \cdots, r)$ cannot vanish identically.

We define further polynomials $P_{i}(x), P_{i}^{(j)}(x)(j=1,2, \cdots, r)$, for $i=1,2, \cdots, r$, by

$$
P_{\imath+1}(x)=P_{i}^{\prime}(x), \quad P_{i+1}^{(j)}(x)=-\lambda_{j} P_{i}^{(j)}(x)+\left\{P_{i}^{(j)}(x)\right\}^{\prime} .
$$

where the accent denotes the derivative with respect to $x$. Next we define

$$
\xi_{i}^{(j)}(x)=P_{i}^{(j)}(x)-e^{\lambda_{j} x} P_{i}(x) \quad(i=0,1, \cdots, r, j=1,2, \cdots, r) .
$$

Then it follows that, for $i=0,1, \cdots, r-1, j=1,2, \cdots, r$

$$
\xi_{i+1}^{(j)}(x)=-\lambda_{j} \xi_{i}^{(j)}(x)+\left\{\xi_{i}^{(j)}(x)\right\}^{\prime} .
$$

From (2) it follows that for $i=0,1,2, \cdots, r$ the lowest possible powers of $x$ in $\xi_{\imath}^{(j)}(x)$ are $x^{m_{j+h-\imath}}$. Therefore, for any positive integer $n$

$$
\left|\xi_{i}^{(j)}\left(t^{-n}\right)\right|_{K} \leqq e^{-n\left(m_{j}+h-i\right)} .
$$

Lastly, we define the determinant $\Delta(x)$ by

$$
\Delta(x)=\left|\begin{array}{cc}
P_{0}(x) & P_{0}^{(1)}(x) \cdots P_{0}^{(r)}(x) \\
P_{1}(x) & P_{1}^{(1)}(x) \cdots P_{1}^{(r)}(x) \\
& \cdots \cdots \cdots \\
P_{r}(x) & P_{r}^{(1)}(x) \cdots P_{r}^{(r)}(x)
\end{array}\right|
$$

From (3) the highest coefficient of the polynomial $\Delta(x)$,

$$
(-1)^{r(r+1) / 2} \lambda_{1} \cdots \lambda_{r} \prod_{1 \leq i<j \leq r}\left(-\lambda_{i}+\lambda_{j}\right) \cdot p p_{1} \cdots p_{r}
$$

is nonzero, where $p, p_{j}(j=1,2, \cdots, r)$ are the highest nonzero coefficients in $P_{0}(x), P_{0}^{(j)}(x)(j=1,2, \cdots, r)$, respectively. Thus $\Delta(x)$ is not identically zero. 


\section{Proof of the Theorem}

Now let $u(t)$ be a polynomial with real coefficients, of degree $k \geqq 0$. And let $u_{j}(t)(j=1,2, \cdots, r)$ be any polynomials with real coefficients. Let

$$
\left|u_{j}(t)-e^{\lambda_{j} / t n} u(t)\right|_{K}=e^{-a,} \quad(\jmath=1,2, \cdots, r) .
$$

By the definition of the valuation, we can consider that all $a$, are positive integers. And also the proof of (1) can be replaced by the proof of following inequality :

$$
k-\sum_{\jmath=1}^{r} a_{\jmath} \geqq-R(n, r) .
$$

There are three cases in the proof.

(I) Suppose that for all integers $j$ with $1 \leqq j \leqq r$

$$
a_{\jmath} \geqq L=L(n, r),
$$

where $L(n, r)$, equation (12) later, is a positive constant depending on only $n$ and $r$.

We use the construction of Section 2 with

$$
m_{j}=\left[\left(a_{j}-L\right) / n\right]+1
$$

for $j=1,2, \cdots, r$, that is

$$
n m_{j}=a_{j}-L+n-\tau,
$$

if $a_{j}-L \equiv \tau, \bmod n\left(0 \leqq \tau_{j} \leqq n-1\right)$.

$E(t)$ is defined by

$$
E(t)=\left|\begin{array}{cccc}
P_{\imath_{1}}\left(t^{-n}\right) & P_{i_{1}}^{(1)}\left(t^{-n}\right) & \cdots & P_{i_{1}}^{(r)}\left(t^{-n}\right) \\
& \cdots \ldots \ldots \ldots . . & \\
& & \\
P_{\imath_{r}}\left(t^{-n}\right) & P_{i_{r}}^{(1)}\left(t^{-n}\right) & \cdots & P_{i_{r}}^{(r)}\left(t^{-n}\right) \\
u(t) & u_{1}(t) & \cdots & u_{r}(t)
\end{array}\right|,
$$

where $i_{1}, \cdots, i_{r}$ are some $r$ distinct numbers chosen from $0,1, \cdots, r$. Since the equality $E(t)=0$ contradicts the fact $\Delta(x) \neq 0$, we may assume that $E(t)$ is not identically zero.

We will compare two estimates for $|E(t)|_{K}$. First, we give the lower estimate. Since for $i=1,2, \cdots, r$ the degrees of $P_{i}(x), P_{i}^{(j)}(x)(j=1,2, \cdots, r)$ are at most $h$,

$$
\left|P_{i}\left(t^{-n}\right)\right|_{K},\left|P_{i}^{(j)}\left(t^{-n}\right)\right|_{K} \geqq e^{-n h} \quad(j=1,2, \cdots, r) .
$$

Therefore, we get 


$$
|E(t)|_{K} \geqq e^{-n r h} .
$$

Next for each integer $\jmath$ with $1 \leqq j \leqq r$, by subtracting the first column multiplied by $e^{\lambda_{j / t^{n}}}$ from the $(j+1)$-th column, we have

$$
E(t)=\left|\begin{array}{cccc}
P_{\imath_{1}}\left(t^{-n}\right) & \xi_{\imath_{1}}^{(1)}\left(t^{-n}\right) & \cdots & \xi_{i_{1}}^{(r)}\left(t^{-n}\right) \\
\cdots \cdots \cdots \cdots \cdots & & \\
P_{\imath_{r}}\left(t^{-n}\right) & \xi_{i_{r}}^{(1)}\left(t^{-n}\right) & \cdots & \xi_{i_{r}}^{(r)}\left(t^{-n}\right) \\
u(t) & u_{1}(t)-e^{\lambda_{1 / t} n} u(t) & \cdots & u_{r}(t)-e^{\lambda_{r} / t} u(t)
\end{array}\right| .
$$

Inequalities (5) and $\left|P_{i}\left(t^{-n}\right)\right|_{K} \leqq 1$ give

$$
|E(t)|_{K} \leqq e^{M},
$$

where

$$
\begin{gathered}
M=\max \left\{k-\sum_{j=1}^{r} a_{j}+r L+\sum_{j=1}^{r} \tau_{j}+\frac{1}{2} n r(r-1)-n r h,\right. \\
\left.\max _{1 \leqq j \leqq r}\left\{\frac{1}{2} n r^{2}-\frac{1}{2} n r-L-\tau_{j}-n r h\right\}\right\} .
\end{gathered}
$$

Now we define

$$
L=L(n, r)=\frac{1}{2} n r^{2}-\frac{1}{2} n r+1 .
$$

Then from (9) and (10), using that $\sum_{j=1}^{r} \tau_{j} \leqq(n-1) r$,

$$
\begin{aligned}
k-\sum_{j=1}^{r} a_{\jmath} & \geqq-r L-\sum_{j=1}^{r} \tau_{j}-\frac{1}{2} n r(r-1) \\
& \geqq-\frac{1}{2} n\left(r^{3}+r\right) .
\end{aligned}
$$

(II) Assuming that for all integers $j$ with $1 \leqq j \leqq r$

clearly,

$$
a_{\jmath} \leqq L-1,
$$

$$
\begin{aligned}
k-\sum_{j=1}^{r} a_{\jmath} & \geqq-r(L-1) \\
& \geqq-\frac{1}{2} n\left(r^{3}+r\right) .
\end{aligned}
$$

(III) Suppose that

$$
a_{1}, \cdots, a_{\kappa} \geqq L, \quad a_{\kappa+1}, \cdots, a_{r} \leqq L-1 \quad(\kappa=1,2, \cdots, r-1) .
$$


If we rearrange $a_{1}, a_{2}, \cdots, a_{r}$, it will be reduced to this case. By the definition (12)

Let $\kappa \geqq 2$. Since

$$
a_{1}, \cdots, a_{\kappa} \geqq L(n, r) \geqq L(n, \kappa) .
$$

$$
k-\sum_{\jmath=1}^{\kappa} a_{\jmath} \geqq-R(n, \kappa) .
$$

therefore,

$$
\begin{aligned}
k-\sum_{j=1}^{r} a_{\jmath} & \geqq-R(n, \kappa)-(r-\kappa)\{L(n, r)-1\} \\
& \geqq-R(n, r) .
\end{aligned}
$$

When $\kappa=1$, by the following Lemma, the same result will hold.

LEMMA. Let $n$ be a positive integer. If $\lambda$ is nonzero real number, and $u(t)$, $v(t)$ are real polynomials with $u(t) \neq 0$, then

$$
|u(t)|_{K}\left|v(t)-e^{\lambda / t^{n}} u(t)\right|_{K} \geqq e^{-n} .
$$

\section{Proof of the Lemma}

Let $u(t)$ be a polynomial with real coefficients, of degree $k \geqq 0$. And let

$$
\left|v(t)-e^{\lambda / t n} u(t)\right|_{K}=e^{-a} .
$$

In order to prove (13), we just need to show $k-a \geqq-n$. We use the construction of polynomials with

$$
h=m-1, \quad m=[(a-1) / n]+1 .
$$

Simply, set $P_{\imath}^{(1)}=Q_{\imath}, u_{1}=v$. Consider the estimation of

$$
E_{1}(t)=\left|\begin{array}{ll}
P_{i}\left(t^{-n}\right) & Q_{i}\left(t^{-n}\right) \\
u(t) & v(t)
\end{array}\right|,
$$

where $i=0$ or 1 . Similarly as the first part of the proof of the Theorem, we can prove (13). Hence

and

$$
\left|E_{1}(t)\right|_{K} \geqq e^{-n h}
$$

$$
\left|E_{1}(t)\right|_{K} \leqq e^{M_{1}},
$$

where $M_{1}=\max \{-a, k-n(m+h-i)\}$.

From the two estimates.

$$
k-n(m+h-i) \geqq-n h .
$$

Therefore, we get the result of Lemma. 
Acknowledgements. We would like to acknowledge the help and attention which has been given to us by the members of our graduation committee. Above all, we must thank our advisor Proof. Takayoshi Mitsui who has provided the guidance, enthusiasm and encouragement necessary to get us through the past few years, and Dr. Masanori Toyoda who has invited us into our studies.

\section{REFERENCES}

[1] H. Davenport and D. J. Lewis, An analogue of a problem of Littlewood, Michigan Math. J. 10 (1963), 157-160.

[2] A. BAKER, On an analogue of Littlewood's diophantine approximation probrem, Michigan Math. J. 11 (1964), 247-250.

Department of Mathematics

GAKUSHUIN UNIVERSITY

1-5-1 MejiRo, Toshima-Ku

TOKYO 171 JAPAN 